$\mathbb{T}$ periodica polytechnica

Civil Engineering

$57 / 1(2013) 11+19$

doi: 10.3311/PPci.2137

http://periodicapolytechnica.org/ci

Creative Commons Attribution (1)

RESEARCH ARTICLE

\section{Overdamped mechanical model of myosin II}

\author{
András Bibó / Mihály Kovács / György Károlyi
}

Received 2012-06-21, accepted 2012-12-13

\begin{abstract}
Due to their small size molecular systems are often overdamped and they are affected by significant fluctuations due to thermal forces. In this paper we investigate the effect of overdamping on a simple mechanical model of myosin II, the motor protein responsible for muscle contraction. We demonstrate that this model, based on the experimentally observed shape of the protein's subdomains, is consistent with the available experimental results. We also shed new light on the debate whether the powerstroke is a sudden conformational change followed by relaxation to equilibrium or a thermal fluctuation followed by a fixation by a ratchet-like mechanism in an energetically favourable conformation: we propose that these mechanisms coexist.
\end{abstract}

\section{Keywords}

Myosin II $\cdot$ muscle model $\cdot$ motor protein

\section{András Bibó}

Institute of Nuclear Techniques, Budapest University of Technology and Economics, Múegyetem rkp. 3, H-1111 Budapest, Hungary

e-mail: biboan@reak.bme.hu

\section{Mihály Kovács}

ELTE-MTA “Momentum” Motor Enzymology Research Group, Department of Biochemistry, Eötvös Loránd University, Pázmány Péter sétány 1/C, H-1117 Budapest, Hungary

\section{György Károlyi}

Institute of Nuclear Techniques, Budapest University of Technology and Economics, Múegyetem rkp. 3, H-1111 Budapest, Hungary

\section{Introduction}

Skeletal muscles show great variety, but their main structure is the same. Repeating functional segments called sarcomeres [1] provide the contraction while the actin and myosin filaments, the main ingredients of sarcomeres, slide beside each other, see Fig. 1. The sliding motion is provided by motor proteins called myosin II [1] that can connect to binding sites along the actin filament forming cross-bridges. Myosin II molecules provide the powerstroke, a force pulling the myosin filament along the actin filament thus shortening the muscle tissue.

The energy required for the contraction is provided by adenosine triphosphate (ATP), when it is hydrolysed, it turns into adenosine diphosphate (ADP) and phosphate ion while energy is liberated. This energy is converted into mechanical energy by myosin II [1].

The main units of myosin II have been observed in experiments. The motor domain binds ATP and attaches to the actin filament, while a lever arm rotates about $70^{\circ}$ around the motor domain providing the powerstroke [2]. Despite the increasing amount of X-ray crystallographic and other observations, the mechanical force and displacement transduction between the different parts of the motor domain are not yet clarified [3]. In a previous paper [4], we proposed a simple mechanical model based on the existence of an internal lever arm within the motor domain to explain the mechanical behaviour of myosin II. This model is capable of explaining the observed four-stroke motion of myosin II [5]. After the detachment of myosin II from the actin filament, due to binding of ATP (first stroke), recovery takes places: the motor protein primes itself for the next powerstroke by positioning the motor domain forward (second stroke). Then myosin II attaches to a binding site on the actin filament (third stroke), finally the powerstroke takes place when the lever arm rotates about $70^{\circ}$ providing contraction (fourth stroke).

Our model in Ref. [4], however, does not include the effects of thermal forces, which are not negligible on the length scale of motor proteins. At the length scale corresponding to myosin II (few tens of nanometers) thermal collisions occur on the picosecond timescale [6]. According to Einstein's relation [7], the diffusion coefficient for the motor domain (taken to be a sphere 


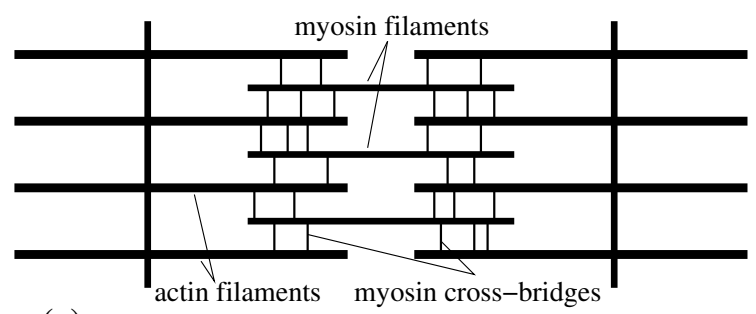

(a)

sarcomere

Fig. 1. Sketch of a sarcomere in (a) resting and in (b) contracted state. Myosin II cross-bridges pull the myosin filaments along the actin filaments,

of radius $R_{M}=2 \mathrm{~nm}$ ) resulting from thermal collisions is

$$
D=\frac{k_{B} T}{6 \pi \eta r}=110 \mathrm{~nm}^{2} / \mu \mathrm{s},
$$

where $k_{B}=0.0138 \mathrm{pN} \cdot \mathrm{nm} / \mathrm{K}$ is the Boltzmann constant, the temperature is $T=300 \mathrm{~K}$, the dynamic viscosity of water is $\eta=0.001 \mathrm{pN} \cdot \mu \mathrm{s} / \mathrm{nm}^{2}[6]$. Visually, myosin II 'visits' a $10 \times 10$ $\mathrm{nm}^{2}$ square in every microsecond, a square whose linear size is five times the size of the motor domain. As the length of the four stroke enzymatic cycle is on the order of $10 \mathrm{~ms}$ [6], thermal forces can move the motor domain to a considerable distance. This shows that thermal forces are indeed important, hence in this paper we extend our previous model [4] by incorporating the effects of random thermal forces.

Recent experimental techniques provide a quite detailed picture of the main elements that build up myosin II [8,9]. Within the motor domain, several subdomains have been revealed, which seem to keep their shape during the four strokes of myosin II motion, but whose relative position seems to be changing. These subdomains include the upper (U50) and lower (L50) sides of the cleft as the parts of the motor domain that attach to the binding site of the actin filament. The U50 and L50 subdomains can move relative to each other, they can open up or close the gap between them depending on the actual phase during the four strokes. Within the motor domain, other subdomains can be identified [8,9], like the $N$-terminal subdomain that seems to serve as a support for all other subdomains, the converter domain providing the large displacement of the lever arm, and the relay helix which we identify as the internal lever arm for our model. It has been observed [2, 9, 10] that the relay helix shows a seesaw motion during the enzymatic cycle, which is closely coupled with the opening and closing of the cleft. Although all these subdomains and others have been clearly identified in experiments, their role is not clearly identified.

Another interesting question, subject of a long-term debate, is the detailed mechanism of the powerstroke. The model of Ref. [11] proposed a thermal ratchet or Kramers mechanism. According to this model, random thermal fluctuations move the molecule to the close vicinity of a desired conformation, where it becomes fixed by a ratchet-like mechanism [6]. Another model, the Eyring mechanism, has been proposed in Ref. [12]. Here, a sudden local change occurs first in the initial conforma-

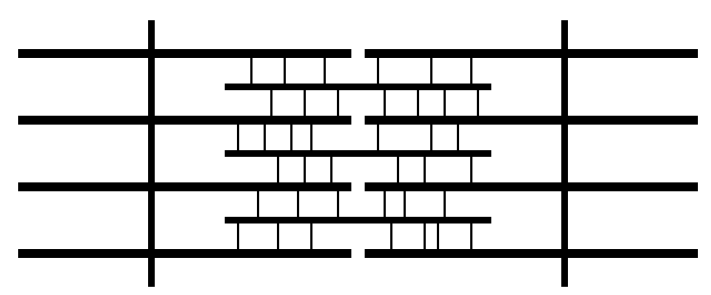

(b)

which results in a shortening of the sarcomere and hence in the contraction of the muscle tissue.

tion, then the whole molecule slowly relaxes into a final equilibrium conformation. Based on our model we speculate that these two mechanisms might act together.

Based on the experimentally observed subdomains of myosin II, in Sec.2 2 we present a low degree of freedom mechanical model for myosin II. After writing the equations of motion in Sec. 3, in Sec. 4 we show that, with a reasonable choice of the parameters but neglecting the thermal fluctuations, the model exhibits the experimentally observed behaviour of myosin II. Finally, conclusions are drawn in Sec.5

\section{Mechanical model of myosin II}

The model we propose, beside the conventional external lever arm, consists of an internal lever arm, similarly to the model proposed in Ref. [4]. This internal lever arm splits the time scales for the motion of the lever arm and the mechanism that binds the motor domain to the actin filament, such time scale splitting device in overdamped systems has been proposed in Ref. [13].

The model, shown in Fig. 2. has three distinct phases according to the relation of the motor domain to the actin filament. The motor domain can either be separated from the actin filament, it can be bound to a binding site on the actin filament, or it can slide along the actin while trying to reach the binding site, see Fig. 2. e). According to the content of the ATP binding site, the model can either be in an excited state after hydrolysing ATP or it can be in a relaxed state before hydrolysing ATP. In our model, this is modelled by spring $k_{H}$, see Fig. 2(a), connecting the internal lever arm to the N-terminal subdomain: when ATP is hydrolyzed, the upper end of this spring is displaced downwards by $\Delta$ with respect to the $\mathrm{N}$-terminal subdomain so that the liberated energy is stored as elastic energy in the system. The upper end is repositioned in a later step of the enzymatic cycle, see below.

The model consists of rigid bodies corresponding to the lower (L50) and upper (U50) sides of the cleft, the N-terminal subdomain, the converter domain, the internal and the external lever arms, connected by springs and hinges (cf. Fig. 2(a)). The rigid parts imitate the observed subdomains of myosin II. The springs provide the storage of energy, the timing of the motion of the rigid parts, and they model the elastic forces within the molecule. There is a special connection between U50 and L50: 

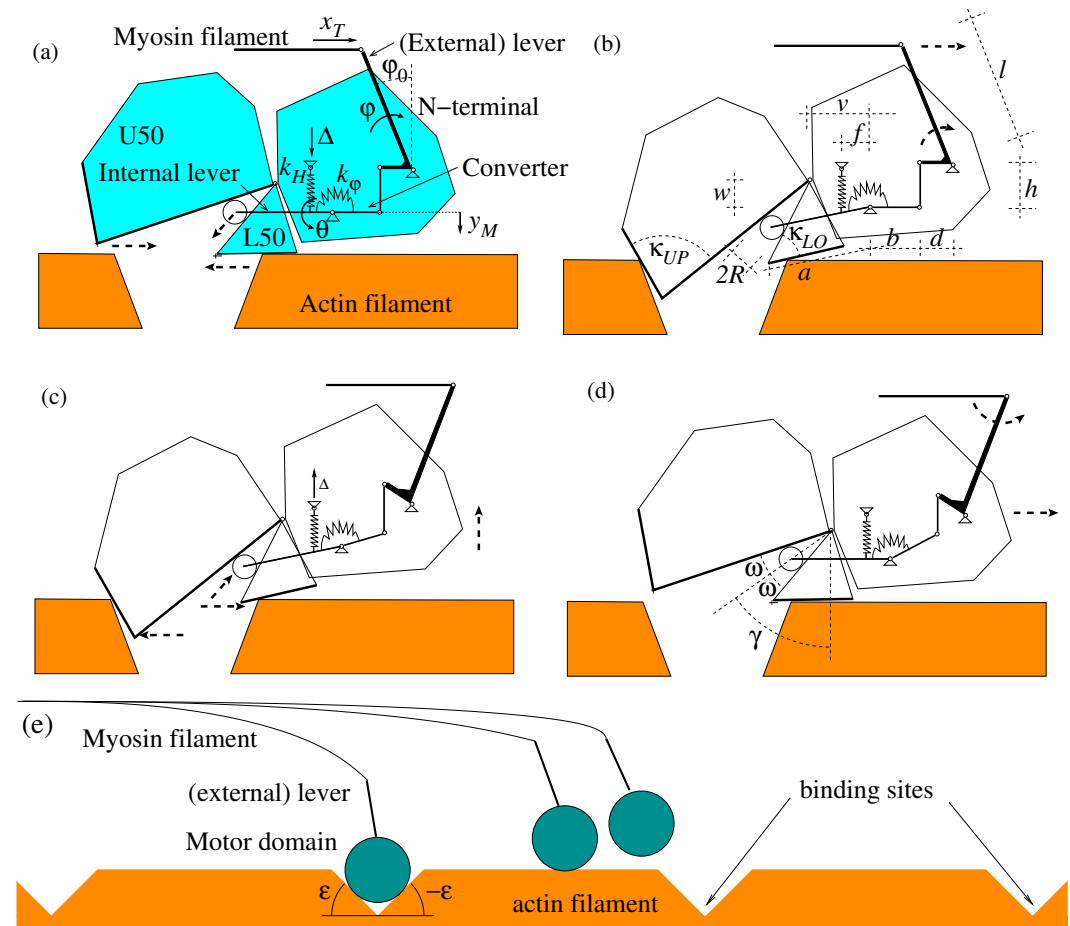

Fig. 2. (a-d) The four strokes of the model of myosin II. The dashed arrows show the direction of motion of the elements during the following stroke. (a) State after the recovery stroke. The names of the rigid elements corresponding to observed subdomains are indicated. The four main variables describing the conformation are also shown. $\Delta$ is the displacement of the upper support of spring $k_{H}$ as a result of ATP hydrolysis. (b) Myosin II bound to the actin fila-

it is a rigid circle that keeps the distance between these subdomains. The center of this circle is attached to the left end of the internal lever arm, its motion is governed by the rotation of the internal lever arm. We assume that this circle can slide without friction inside the cleft, but always remains in contact with the U50 and L50 subdomains. This circle imitates the effect of a water droplet inside the cleft, whose existence we assume based on observations [9]. Due to the hydrophobicity of myosin II it tries to reduce its surface exposed to water molecules, hence the cleft favors being closed. In our model this is accounted for by the permanent contact of the water droplet at the end of the internal lever arm with the U50 and L50 subdomains. The same hydrophobic property of myosin II causes in our model a permanent force downward, which takes into account that myosin II favors binding to the actin filament to reduce its surface exposed to water.

We assume that the $\mathrm{N}$-terminal subdomain has no rotational degree of freedom. This is a reasonable assumption because its orientation is always restored upon binding to the actin filament. Omitting this degree of freedom, the position of the system can be described by four variables. As a convenient choice, we use the following variables to describe the possible configurations (see Fig. 2(a)):

$y_{M}$ is the vertical position of the N-terminal subdomain, positive downwards, and it is zero before the first stroke, before ATP is hydrolyzed; ment. The length of various distances are also indicated. (c) Post-powerstroke conformation. (d) Detached conformation before recovery stroke. (e) Geometric constraint modelling binding sites along the actin filament. Motor domains (full circles) can be bound to a binding site (valley), can slide along the actin filament, or can be detached from actin.

$x_{T}$ is the horizontal displacement of the upper end of the (external) lever arm, it is positive in the direction of the sliding motion, and is zero at the start of the motion;

$\theta$ is the rotation of the internal lever arm with respect to the horizontal direction, and is positive counter-clockwise;

$\varphi$ is the rotation of the (external) lever arm with respect to the state before ATP hydrolysis, it is positive clockwise.

As large molecules like myosin II are overdamped, the damping forces are orders of magnitudes higher than inertial forces. Hence we assume that inertial forces are negligible [4, 13], which results in first order differential equations for the displacement variables $y_{M}, x_{T}, \theta$ and $\varphi$.

According to the fluctuation-dissipation theorem [14], a micromolecular system is subject to random thermal forces of Gaussian distribution with a variance $\sigma_{i}^{2}=c_{i} k_{B} T / \Delta t$, where $c_{i}$ is the damping coefficient of the $i$ th degree of freedom and $\Delta t$ is the length of a chosen time interval. In our model, this random force accompanies any damping force.

On a qualitative level, the behaviour of the model consists of the following main steps, the four strokes:

ATP hydrolysis and actin binding. Figure 2(a) shows the model before this first stroke. During this stroke the whole myosin is carried along the actin filament by the other myosin II molecules being in their powerstroke phase. While sliding 
along the actin filament random thermal fluctuations are applied to the upper end of spring $k_{H}$ until the elastic energy stored in the compressed spring reaches $25 k_{B} T$ corresponding to the energy released from ATP. In this instant, the upper end of the spring is fixed in this high energy state, which forces the internal level to rotate counter clockwise, which, in turn, decreases the cleft by the displacement of the water droplet at the end of the internal lever arm while U50 and L50 also suffer a counter clockwise rotation. The decrease of the cleft allows for a conformation suitable for actin binding: the U50 and L50 subdomains can now fit into the valleys of Fig. 2(e) corresponding to the binding sites. When the U50 and L50 subdomains reach a binding site, binding occurs, this ends this stroke. The conformation ending this phase is shown in Fig. 2(b).

Actin-bound state initiating the powerstroke. During the previous stroke, due to the rotation of the internal lever arm, the rotational spring at the right end of the internal lever becomes strained. The moment applied by this rotational spring, via the converter domain, on the external lever arm results in the powerstroke: the external lever arm rotates clockwise. This displacement pulls the myosin filament attached to the top of the external lever arm, and hence results in the sliding motion of the actin and myosin filaments with respect to each other leading to the contraction of the muscle fiber. This stroke ends in the post-powerstroke conformation, shown in Fig.2(c), when the rotational spring is no longer strained.

ATP binding and detachment from actin. Under physiological conditions, ATP is available in abundance. Hence we assume that in the post-powerstroke state the previously hydrolysed ADP is released and a fresh ATP is obtained immediately. In our system this is modelled by restoring the upper end of spring $k_{H}$ to its original location on the N-terminal subdomain. This provides an upward force on the internal lever arm and the U50 and L50 subdomains, so the myosin II molecule detaches from the actin filament quite rapidly. With the detachment, this stroke ends, and the pre-recovery stroke conformation is reached, see Fig. 2(d).

Recovery-stroke The clockwise rotation of the internal lever arm results in opposite strain in the rotational spring, hence the external lever arm starts to rotate counter clockwise. Because the myosin molecule is no longer bound to the actin filament, this rotation occurs around the upper end, which means that the myosin II molecule swings forward with respect to the actin filament in search for a new binding site. When the original conformation is reached, the whole enzymatic cycle starts over with the hydrolysis of the new ATP.

\section{Equations of motion}

The forces acting on each subdomain are shown in Fig. 3 , Let us denote the position of the central point of the (external) lever arm by

$$
x_{L}=x_{T}+\frac{1}{2} \ell \sin \left(\varphi_{0}-\varphi\right), \quad y_{L}=y_{M}-h-\frac{1}{2} \ell \cos \left(\varphi_{0}-\varphi\right),
$$

where $\varphi_{0}$ is the angle of the external lever arm with the vertical direction in the initial state shown in Fig. 2(a), $\ell$ is the length of the external lever arm, and $h$ is the vertical distance between the two hinged supports on the N-terminal subdomain, see Fig. 2(b). The velocity components of the central point of the (external) lever arm are

$$
\dot{x}_{L}=\dot{x}_{T}-\dot{\varphi} \frac{1}{2} \ell \cos \left(\varphi_{0}-\varphi\right), \quad \dot{y}_{L}=\dot{y}_{M}-\dot{\varphi} \frac{1}{2} \ell \sin \left(\varphi_{0}-\varphi\right) .
$$

We assume the external lever arm to be cylindrical, and for computing the damping forces we need the velocity components in the parallel $\left(v_{\|}\right)$and in the perpendicular $\left(v_{\perp}\right)$ direction with respect to the direction of the (external) lever arm:

$$
\begin{aligned}
& v_{\|}=\dot{x}_{L} \sin \left(\varphi_{0}-\varphi\right)+\dot{y}_{L} \cos \left(\varphi_{0}-\varphi\right), \\
& v_{\perp}=\dot{x}_{L} \cos \left(\varphi_{0}-\varphi\right)-\dot{y}_{L} \sin \left(\varphi_{0}-\varphi\right) .
\end{aligned}
$$

This way we can write the damping forces and moment acting on the lever arm, they are, according Stokes' law, $c_{\|} v_{\|}, c_{\perp} v_{\perp}$ and $c_{\varphi} \dot{\varphi}$, respectively, where $c_{\|}, c_{\perp}$ and $c_{\varphi}$ are the corresponding damping coefficients.

We assume that the displacements of the converter domain are small, which implies that the vertical rod between the lever arm and the converter domain always remains vertical. Similarly, we also assume that the horizontal projection of distances $b, d, f$ and $v$ (Fig. 2(b)) always remain the same during the motion.

Now we can write the moment equation of the external lever arm around the intersection point of the line of action of forces $D_{y}$ and $F$, see Fig. 3

$$
\begin{aligned}
\left(c_{\|} v_{\|}\right. & \left.+R_{\|}\right) \frac{\ell}{2} \sin \left(2 \varphi_{0}-2 \varphi\right)+\left(c_{\perp} v_{\perp}+R_{\perp}\right) \frac{\ell}{2} \cos \left(2 \varphi_{0}-2 \varphi\right) \\
& -c_{\varphi} \dot{\varphi}-M+D_{x} \ell \cos \left(\varphi_{0}-\varphi\right)-S d=0,
\end{aligned}
$$

where $R_{\|}$and $R_{\perp}$ are random forces, $M$ is random moment of Gaussian distribution and zero mean corresponding to thermal fluctuations, $d$ is the length of the lower part of the external lever arm (see Fig. 2(b)), which is assumed to be constant due to the assumption of small displacements. Note that no inertial forces appear due to overdamping. From the balance of the horizontal forces on the union of the converter domain, the N-terminal, the internal lever arm, the U50 and L50 subdomains we find the internal reaction force $D_{x}$ as

$$
D_{x}=c_{M x} \dot{x}_{M}+R_{M x}-F_{U P} \sin \epsilon_{U P}-F_{L O} \sin \epsilon_{L O},
$$

where $x_{M}=x_{T}+\ell \sin \left(\varphi_{0}-\varphi\right)-d-b$ is the position of the Nterminal subdomain with $b+d$ as the constant horizontal length of the converter domain,

$$
c_{M x} \dot{x}_{M}=c_{M x}\left[\dot{x}_{T}-\dot{\varphi} \ell \cos \left(\varphi_{0}-\varphi\right)\right]
$$

is the horizontal damping force on the union of the converter domain, the N-terminal, the internal lever arm, the U50 and L50 


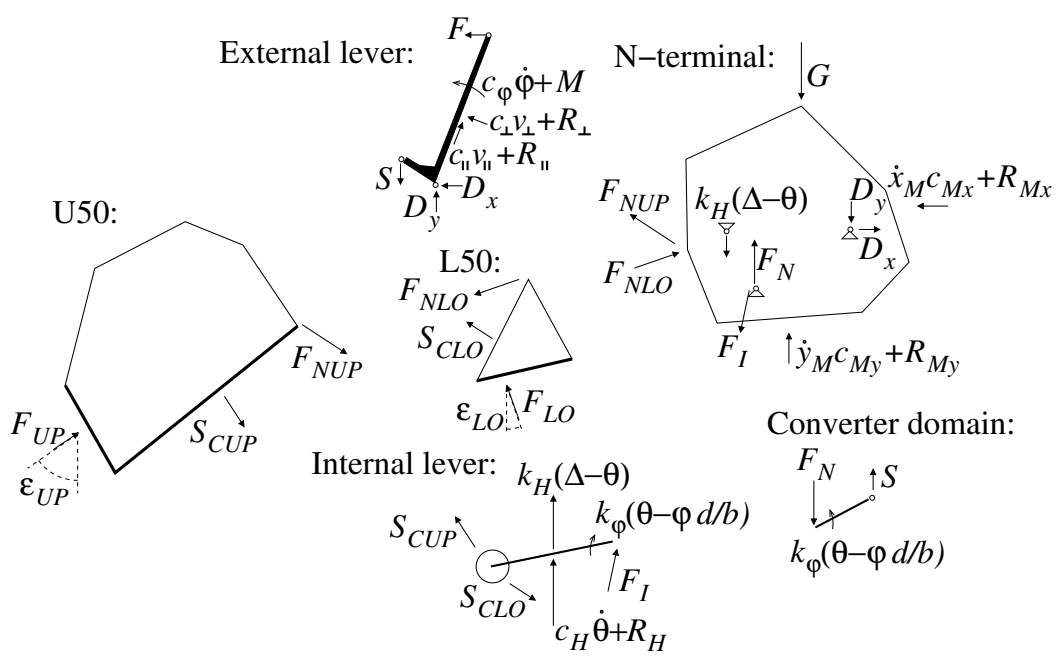

Fig. 3. Free-body diagram: forces acting on the subdomains of myosin II.

subdomains, $R_{M x}$ is the thermal force (Gaussian with zero mean) acting on the N-terminal subdomain, $F_{U P}$ and $F_{L O}$ are the reaction forces arising from the contact between U50 and L50, respectively, and the valleys of slope $\epsilon_{U P}$ and $\epsilon_{L O}$, see Fig. 3 Force $S$, which is always vertical due to the assumption of small displacements within the motor domain, can be computed from the balance of moments on the converter domain:

$$
S=\frac{k_{\varphi}(\varphi d / b-\theta)}{b}
$$

where $k_{\varphi}$ is the stiffness of the rotational spring, and $\varphi d / b$ is the rotation of the converter domain.

Let us now express the half angle of the cleft (Fig.2(d)) as

$$
\omega=\arcsin \frac{R}{L}
$$

where $R$ is the radius of the water droplet in the clefts, and $L$ is the distance of the center of the droplet from the hinge connecting U50 and L50:

$$
L=\sqrt{(a \cos \theta-v)^{2}+(a \sin \theta+w)^{2}}
$$

with $a$ as the length of the internal lever arm, for distances $v$ and $w$ see Fig. 2(b). The line of length $L$ connecting the center of the water droplet to the hinge between U50 and L50 has an angle $\gamma$ with the vertical (cf.2(d)):

$$
\gamma=\arctan \frac{a \cos \theta-v}{a \sin \theta+w} .
$$

Using these angles, we write the balance of moments acting on the internal lever arm around its right end:

$$
\begin{aligned}
& k_{H}(\theta f-\Delta) f+c_{H} \dot{\theta} f+R_{H} f+k_{\varphi}(\theta-\varphi d / b) \\
& \quad+S_{C U P}[\sin (\omega+\gamma) a \cos \theta+\cos (\omega+\gamma) a \sin \theta] \\
& \quad-S_{C L O}[\cos (\gamma-\omega) a \sin \theta+\sin (\gamma-\omega) a \cos \theta]=0,
\end{aligned}
$$

where $f$ is the constant horizontal distance between the right end of the internal lever arm and the attachment point of spring $k_{H}$, $c_{H}$ is the damping coefficient for the rotation of the internal lever arm, $R_{H}$ is the Gaussian random thermal force of zero mean acting on the internal lever arm. Forces $S_{C U P}$ and $S_{C L O}$ are internal reaction forces from U50 and L50, respectively, and can be computed from the balance of moments acting on the U50 and L50 subdomains as

$$
\begin{gathered}
S_{C U P}=\frac{F_{U P} \cos \epsilon_{U P} \Delta x_{U P}-F_{U P} \sin \epsilon_{U P} \Delta y_{U P}}{L \cos \omega}, \\
S_{C L O}=\frac{F_{L O} \cos \epsilon_{L O} \Delta x_{L O}-F_{L O} \sin \epsilon_{L O} \Delta y_{L O}}{L \cos \omega},
\end{gathered}
$$

where $\Delta x_{U P}, \Delta y_{U P}$ are the relative horizontal and vertical coordinates, respectively, with respect to the hinge connecting U50 to L50 of the contact point of U50 and the valley on the actin filament, while $\Delta x_{L O}, \Delta y_{L O}$ are the corresponding coordinates for L50. These values can be computed in every case, they depend on the geometry of U50, L50 and the valley. On U50, we need to introduce angle $\kappa_{U P}$, on L50, $\kappa_{L O}$, see Fig. 2(b), to carry out these computations.

The balance of the horizontal and vertical force components acting on all subdomains of the motor domain can be written, respectively, as

$$
\begin{gathered}
-\dot{x}_{M} c_{M x}-R_{M x}-\left(c_{\|} v_{\|}+R_{\|}\right) \sin \left(\varphi_{0}-\varphi\right) \\
-\left(c_{\perp} v_{\perp}+R_{\perp}\right) \cos \left(\varphi_{0}-\varphi\right) \\
+F_{U P} \sin \epsilon_{U P}+F_{L O} \sin \epsilon_{L O}-F=0, \\
G-\dot{y}_{M} c_{M y}-R_{M y}-\left(c_{\|} v_{\|}+R_{\|}\right) \cos \left(\varphi_{0}-\varphi\right) \\
+\left(c_{\perp} v_{\perp}+R_{\perp}\right) \sin \left(\varphi_{0}-\varphi\right) \\
-F_{U P} \cos \epsilon_{U P}-F_{L O} \cos \epsilon_{L O}=0,
\end{gathered}
$$

where $c_{M x}$ and $c_{M y}$ are, respectively, the horizontal and vertical damping coefficients for the motor domain, $R_{M x}$ and $R_{M y}$ are random thermal forces of Gaussian distribution with zero mean acting on the N-terminal subdomain, and $G$ is the constant vertical load that forces the motor domain towards the actin filament in order to minimize the surface exposed to water molecules as a consequence of the hydrophobicity of myosin II. 
We can combine Eqs. 5[11/14 15 so that it contains only the unknown variables in this following form:

$$
\left[\begin{array}{cccc}
c_{x x} & c_{x y} & c_{x \varphi} & 0 \\
c_{y x} & c_{y y} & c_{y \varphi} & 0 \\
c_{\varphi x} & c_{\varphi y} & c_{\varphi \varphi} & 0 \\
0 & 0 & 0 & c_{\theta \theta}
\end{array}\right]\left[\begin{array}{c}
\dot{x}_{T} \\
\dot{y}_{M} \\
\dot{\varphi} \\
\dot{\theta}
\end{array}\right]=\left[\begin{array}{c}
q_{x} \\
q_{y} \\
q_{\varphi} \\
q_{\theta}
\end{array}\right],
$$

where the elements of the damping matrix are

$$
\begin{gathered}
c_{x x}=-c_{M x}-c_{\|} \sin ^{2}\left(\varphi_{0}-\varphi\right)-c_{\perp} \cos ^{2}\left(\varphi_{0}-\varphi\right), \\
c_{x y}=c_{y x}=\sin \left(\varphi_{0}-\varphi\right) \cos \left(\varphi_{0}-\varphi\right)\left(c_{\perp}-c_{\|}\right), \\
c_{x \varphi}=c_{\varphi x}=c_{\|} \frac{\ell}{2} \sin \left(2 \varphi_{0}-2 \varphi\right) \sin \left(\varphi_{0}-\varphi\right) \\
+c_{\perp} \frac{\ell}{2} \cos \left(2 \varphi_{0}-2 \varphi\right) \cos \left(\varphi_{0}-\varphi\right)+c_{M x} \ell \cos \left(\varphi_{0}-\varphi\right), \\
c_{y y}=-c_{M y}-c_{\|} \cos ^{2}\left(\varphi_{0}-\varphi\right)-c_{\perp} \sin ^{2}\left(\varphi_{0}-\varphi\right), \\
c_{y \varphi}=c_{\varphi y}=c_{\|} \frac{\ell}{2} \sin \left(2 \varphi_{0}-2 \varphi\right) \cos \left(\varphi_{0}-\varphi\right) \\
-c_{\perp} \frac{\ell}{2} \cos \left(2 \varphi_{0}-2 \varphi\right) \sin \left(\varphi_{0}-\varphi\right), \\
c_{\varphi \varphi}=-c_{\varphi}-c_{\|} \frac{\ell^{2}}{4} \sin ^{2}\left(2 \varphi_{0}-2 \varphi\right) \\
-c_{\perp} \frac{\ell^{2}}{4} \cos ^{2}\left(2 \varphi_{0}-2 \varphi\right)-c_{M x} \ell^{2} \cos ^{2}\left(\varphi_{0}-\varphi\right), \\
c_{\theta \theta}=-f c_{H} .
\end{gathered}
$$

The elements of the vector on the right-hand side are

$$
\begin{aligned}
q_{x} & =F+R_{M x}+R_{\|} \sin \left(\varphi_{0}-\varphi\right)+R_{\perp} \cos \left(\varphi_{0}-\varphi\right) \\
& -F_{U P} \sin \epsilon_{U P}-F_{L O} \sin \epsilon_{L O}, \\
q_{y}= & -G+R_{M y}+R_{\|} \cos \left(\varphi_{0}-\varphi\right)-R_{\perp} \sin \left(\varphi_{0}-\varphi\right) \\
& +F_{U P} \cos \epsilon_{U P}+F_{L O} \cos \epsilon_{L O}, \\
q_{\varphi}= & -R_{\|} \frac{\ell}{2} \sin \left(2 \varphi_{0}-2 \varphi\right)-R_{\perp} \frac{\ell}{2} \cos \left(2 \varphi_{0}-2 \varphi\right)+M \\
+ & \left(-R_{M x}+F_{U P} \sin \epsilon_{U P}+F_{L O} \sin \epsilon_{L O}\right) \ell \cos \left(\varphi_{0}-\varphi\right) \\
+ & k_{\varphi}\left(\varphi \frac{d}{b}-\theta\right) \frac{d}{b}, \\
q_{\theta} & =-f k_{H} \Delta+\theta\left(k_{\varphi}+f k_{H}\right)-\varphi k_{\varphi} \frac{d}{b} \\
& +S_{C U P}[\sin (\omega+\gamma) a \cos \theta+\cos (\omega+\gamma) a \sin \theta] \\
& -S_{C L O}[\cos (\gamma-\omega) a \sin \theta+\sin (\gamma-\omega) a \cos \theta] .
\end{aligned}
$$

Note that both the damping matrix and the vector on the righthand side depend on the actual values of the variables. Also note that the values of internal reaction forces $F_{U P}$ and $F_{L O}$ are zero in the detached state, however, they are to be calculated when myosin II is attached to actin. In this latter case, the contact between the actin valley and U50 or L50 is modelled by an elastic force whose magnitude is proportional to the overlap between the contacting members, the proportionality constant is $k_{\text {actin }}$. The direction of the force is perpendicular to the surface of the contacting member penetrated by the corner of the other member. With this choice, we can use the same equations given above both in case of attached and detached states, only the values of $F_{U P}$ and $F_{L O}$ have to be taken care of.

\section{Results}

\subsection{Parameter values}

\subsubsection{Parameters from experimental observations}

As a first step, let us choose suitable values for the parameters of the system. Several geometrical parameters can be obtained from experimental observations [1,6]. These include the length of the external lever $\operatorname{arm} \ell=8 \mathrm{~nm}$ and its initial angle $\varphi_{0}=40^{\circ}$. The radius of the external lever arm is $R_{L}=1.5 \mathrm{~nm}$, that of the motor domain is $R_{M}=2 \mathrm{~nm}$. Data from the Protein Data Bank [15], obtained from crystallographic measurements, allows for the estimation of the following parameters: $a=2.647 \mathrm{~nm}, b=$ $1.503 \mathrm{~nm}, d=0.432 \mathrm{~nm}, h=1.5 \mathrm{~nm}, v=1.658 \mathrm{~nm}, w=$ $0.781 \mathrm{~nm}$, cf. Fig. 2(b). The lengths of the side of U50 and L50 in contact with the water droplet can also be obtained, for U50 we find $4.923 \mathrm{~nm}$, for L50 $2.398 \mathrm{~nm}$, while the radius of the water droplet in the clefts is $R=0.35 \mathrm{~nm}$ [8]. From observed conformation [8] we set $\kappa_{L O}=35.58^{\circ}$ and $\kappa_{U P}=67.83^{\circ}$.

Following Ref. [6], we can calculate the damping coefficients for the external lever arm as

$$
\begin{gathered}
c_{\|}=\frac{2 \pi \eta \ell}{\ln \left(\ell / 2 R_{L}\right)-0.2}=6.44 \cdot 10^{-2} \frac{\mathrm{pN} \cdot \mu \mathrm{s}}{\mathrm{nm}}, \\
c_{\perp}=\frac{4 \pi \eta \ell}{\ln \left(\ell / 2 R_{L}\right)+0.84}=5.52 \cdot 10^{-2} \frac{\mathrm{pN} \cdot \mu \mathrm{s}}{\mathrm{nm}}, \\
c_{\varphi}=\frac{\pi \eta \ell^{3} / 3}{\ln \left(\ell / 2 R_{L}\right)-0.66}=1.67 \mathrm{pN} \cdot \mu \mathrm{s} \cdot \mathrm{nm},
\end{gathered}
$$

where the shape of the lever arm was approximated as a cylinder, and $\eta=10^{-3} \mathrm{~Pa} \cdot \mathrm{s}$ is the dynamic viscosity of water [6]. Similarly, the damping coefficient for the motor domain is

$$
c_{M x}=6 \pi \eta R_{M}=3.77 \cdot 10^{-2} \frac{\mathrm{pN} \cdot \mu \mathrm{s}}{\mathrm{nm}},
$$

where the shape of the motor domain is approximated by a sphere.

\subsubsection{Estimated parameters}

For the geometry of the valleys on the actin filament, we take the width of the valley to be $4.96 \mathrm{~nm}$ with a slope of $\tan \epsilon=$ 2.901, resulting in a depth of $7.2 \mathrm{~nm}$ of the valley. This choice ensures that the width is wide enough so that U50 and L50 can fit in closed state and cannot fit in open state. The slope is in the 


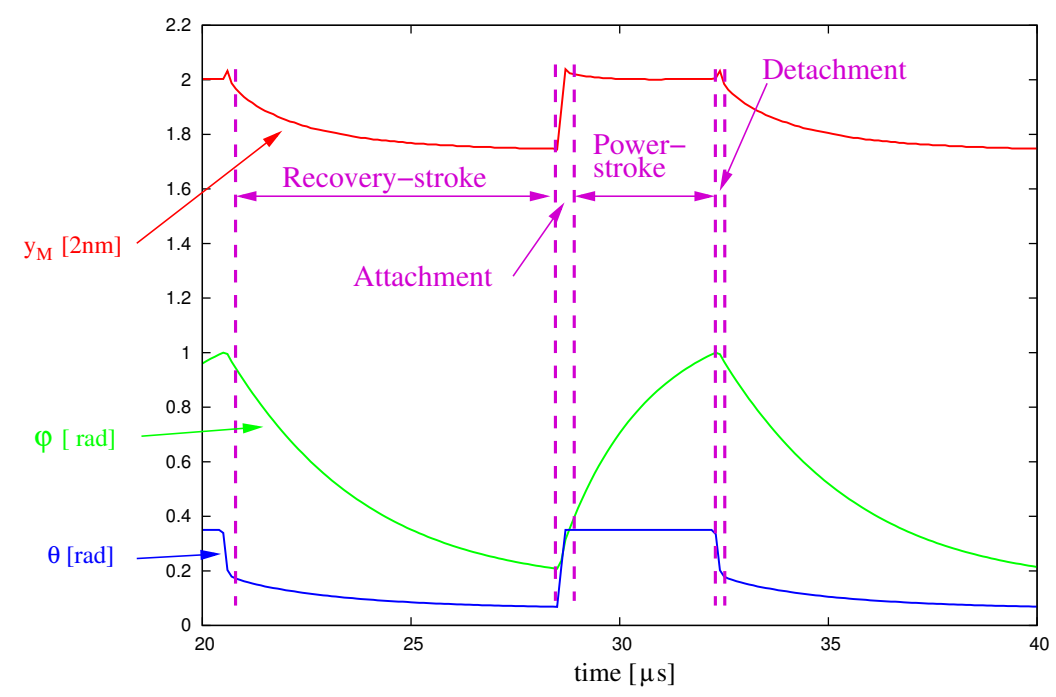

Fig. 4. One enzymatic cycle shown via variables $y_{M}, \varphi$ and $\theta$. The load applied at the end of the myosin filament was $F=0.2 \mathrm{pN}$. Note that $y_{M}$ is

measured in units of $2 \mathrm{~nm}$ so that all curves are close to each other in one figure.

range where the U50 and L50 subdomains can bind and unbind also. The choice of $12 \mathrm{~nm}$ as the initial distance between the right endpoint of the internal lever arm from the bottom of the valley (binding site) on the actin filament ensures that U50 and L50 can slide along the plateau of the actin filament, but they can bind at the valleys due to the downward force $G$.

The damping coefficient $c_{H}$ of the internal lever arm always appears multiplied by $f$, their product is chosen to be $f c_{H}=1.2 \mathrm{pN} \cdot \mu \mathrm{s} / \mathrm{nm}$, this value is found to result in a mean enzymatic cycle length of $20 \mathrm{~ms}$, close to the experimentally observed value [6]. The disposition of the endpoint of spring $k_{H}$ as a consequence of ATP hydrolysis is set as $\Delta=0.7 \mathrm{~nm}$, this value is found to be consistent with the free energy profile of myosin II: with this choice no energy is injected into the system when the upper end of spring $k_{H}$ is restored to its original position in the post-powerstroke conformation. The stiffness parameters are set using data on the free-energy profile: at the hydrolysis of ATP, the liberated energy of $25 k_{B} T$ can be stored in spring $k_{H}$ if $f k_{H} \Delta^{2} / 2=25 k_{B} T$, that is, if $f k_{H}=400 \mathrm{pN} / \mathrm{nm}$. The choice of $k_{\varphi}=240 \mathrm{pN} \cdot \mathrm{nm}$ ensures the experimentally observed $3 k_{B} T$ free energy difference during the powerstroke. The choice of $G=84 \mathrm{pN}$ is consistent with the $15 k_{B} T$ binding energy of myosin II to actin. The elastic constant between the binding valley and U50 or L50 is taken as $k_{\text {actin }}=120 \mathrm{pN} / \mathrm{nm}$, which is high enough to prevent large overlap between the contacting elements, but sufficiently low to avoid numerical fluctuations.

The equations are solved using the Euler method with small fixed time steps of $\Delta t_{E}=1 / 30 \mathrm{~ns}$, and new random forces are generated after every $\Delta t=1 \mathrm{~ns}$ time interval. For larger $\Delta t$ the random forces would average to zero during one timestep (see the discussion on the fluctation-dissipation theory in Section 2), for smaller $\Delta t$ the simulations would take longer. This value was found to be optimal. Also, the value of $\Delta t_{E}$ is set to the maximum for reliable numerical results.

\subsection{Numerical simulations}

In this paper, we investigate the system without the random thermal forces and hundred times magnified damping coefficients. This way we can investigate the deterministic behaviour of the system, the numerical results obtained with fluctuations are going to be published in a forthcoming paper. In Fig. 4 we see one enzymatic cycle of the model. The system consumes one ATP in each cycle, when energy is introduced into the system via spring $k_{H}$.

The four strokes can clearly be identified, as indicated in Fig. 4. The elevated plateaus on the $y_{M}$ curve belong to the powerstroke, during which the motor domain is bound to actin. During this interval the external lever arm has an increasing rotation $\varphi$, the powerstroke is performed. During the recovery stroke, both $y_{M}$ and $\varphi$ decrease, myosin II returns into its initial state, detached from actin to prepare for the next ATP hydrolysis. These two main intervals are separated by short intervals of attachment and detachment. During these short intervals, rotation $\theta$ of the internal lever arm changes rapidly.

Note that the length of the cycle in this experiment is around $10 \mu \mathrm{s}$, much shorter than that for real myosin II, which is around $20 \mathrm{~ms}$ [6]. This is the consequence of the lack of thermal noise, because in lack of thermal noise the model does not need to wait for the hydrolysis to occur, it happens immediately, hence the motion is much faster. Switching thermal noise on with the parameters as identified in the previous subsection, we measure periods of varying length between $2 \mathrm{~ms}$ and $39 \mathrm{~ms}$, with an average of $20 \mathrm{~ms}$. Obviously, due to the random thermal fluctuations, the strict periodicity of the behavior is lost in that case, but this is the realistic behavior.

With the increase of the load $F$ at the end of the myosin filament, one expects that there exists a threshold load value when the myosin II molecule can be torn off the actin filament. This threshold value has been measured to exceed $10 \mathrm{pN}[6]$. We 


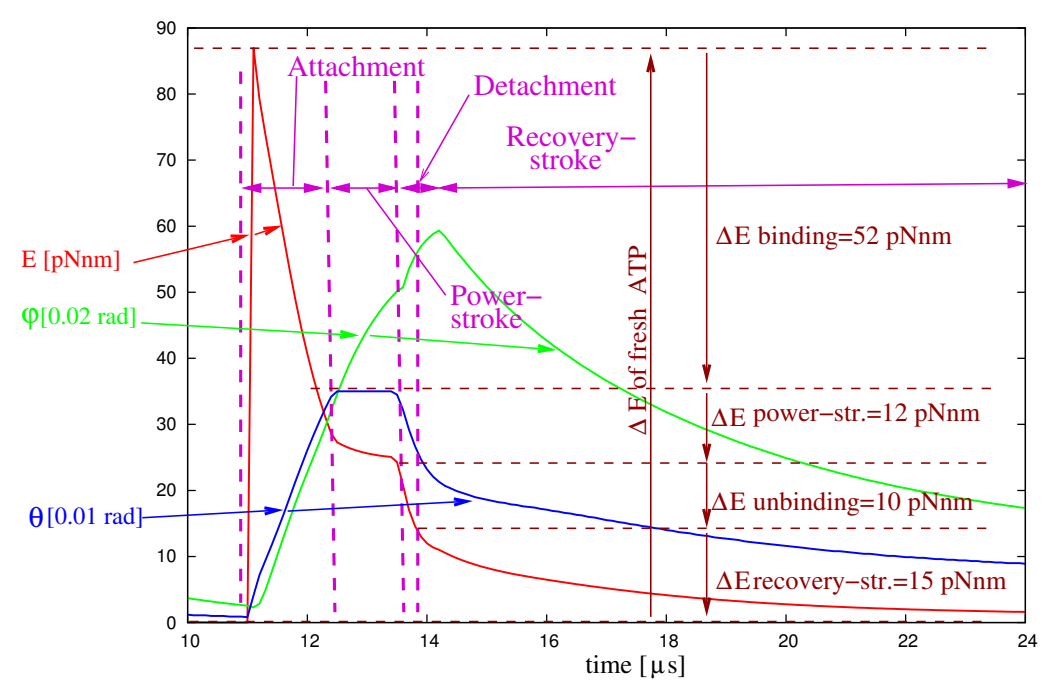

Fig. 5. Energy $(E)$ profile in the model during motion. Arrows indicate the energy differences between the various phases. For reference, the rotations of

carry out a numerical simulation where force $F$ is increased monotonically, while myosin II is bound to actin. The load is increased from zero to $50 \mathrm{pN}$ in $300 \mu$ s, and we found that the motor domain is pulled off the actin filament around $30 \mathrm{pN}$, which is a realistic value not contradicting the experimental observations.

The free energy stored in the system during motion can also be measured, see Fig. 5, in case of no random forces. In our model, we measure free energy as the elastic energy stored in the springs. Figure 5 gives realistic energy differences between the various strokes of the enzymatic cycle. We find that the maximum free energy in the system corresponds to around $90 \mathrm{pN} \cdot \mathrm{nm}$, which is not far from the experimentally observed $25 k_{B} T \approx 100 \mathrm{pN} \cdot \mathrm{nm}$, the energy liberated during ATP hydrolysis. The free energy difference between the unbound and the strongly bounded states of myosin II to actin is around $15 k_{B} T \approx 60 \mathrm{pN} \cdot \mathrm{nm}[6]$, in accordance with the value shown in Fig. 5. The free-energy difference that can be measured during lever arm rotation is around $3 k_{B} T \approx 12 \mathrm{pN} \cdot \mathrm{nm}[16]$; this is also consistent with our numerical simulation.

\section{Conclusions}

In this paper we have presented a simple, low degree of freedom system to model the behavior of myosin II, the motor protein responsible for muscle contraction. This model includes realistic geometrical parameters with estimated damping and stiffness values, and the effects of random thermal forces are also incorporated. Based on numerical simulations neglecting the effects of thermal fluctuations we find that the model shows a very realistic behaviour in accordance with experimental observations: it performs the four-stroke enzymatic cycle using the energy of one ATP in each cycle, and the free energy profile of the system is also consistent with measured data. Similar good agreement is found when thermal fluctuations are turned on, but the external $(\varphi)$ and the internal $(\theta)$ lever arms are also shown.

those results are to be published elsewhere.

We speculate that the debate on the details of the powerstroke, whether it is a Kramers or an Eyring mechanism, can also be resolved by our model. The Eyring mechanism is present in our model, since the hydrolysis of ATP causes a sudden change in the conformation of the system by suddenly changing the position of the internal lever arm (see Fig. 4). This is followed by a longer relaxation of the system to a new equilibrium conformation. However, a Kramers mechanism is also present in our model, because the thermal fluctuations can move the myosin II molecule close to a binding site by pulling along the myosin filament at the upper end of the external lever arm. When close to the binding site, the motor domain can attach to the binding site thus providing a ratchet-like mechanism. This allows us to speculate that in real myosin II molecules the two mechanisms coexist. We intend to more thoroughly investigate this idea in a future publication.

\section{Acknowledgements}

Financial support from OTKA under grant no. K 100894 is hereby gratefully acknowledged. We are indebted for the valuable discussions with T. Tarnai and P. Várkonyi.

\section{References}

1 Bagshaw CR, Muscle contraction., Kluwer Academic Publishers, Dordrecht, 1993.

2 Fischer S, Windshügel B, Horak D, Holmes KC, Smith JC, Structural mechanism of the recovery stroke in the Myosin molecular motor, Proc. Nat. Acad. Sci. (USA), 102, (2005), 6873-6878, DOI 10.1073/pnas.0408784102.

3 Houdusse A, Sweeney HL, Myosin motors: missing structures and hidden springs, Curr. Opin. Struct. Biol., 11, (2001), 182-194, DOI 10.1016/S0959440X(00)00188-3

4 Bibó A, Kovács M, Károlyi G, Internal lever arm model for myosin II., IUTAM symposium on dynamics modeling and interaction control in virtual and real environments, (Budapest, Hungary, 2010), In: Stépán G, Kovács LL, Tóth A (eds.), IUTAM bookseries, Springer, 2011, pp. 155-163. 
5 Lymn RW, Taylor EW, Mechanism of adenosine triphosphate hydrolysis by actomyosin, Biochemistry, 10, (1971), 4617-4624, DOI 10.1021/bi00801a004.

6 Howard J, Mechanics of motor proteins and the cytoskeleton, Sinauer, Sunderland, 2001.

7 Einstein A, Über die von der molekularkinetischen Theorie der Wärme geforderte Bewegung von in ruhenden Flüssigkeiten suspendierten Teilchen, Annalen der Physik, 322, (1905), 549-560, DOI 10.1002/andp.19053220806. In German.

8 Yang Y, Gourinath S, Kovács M, Nyitray L, Reutzel R, Himmel DM, O'Neall-Hennessey E, Reshetnikova L, Szent-Györgyi AG, Brown JH, Cohen C, Rigor-like structures from muscle myosins reveal key mechanical elements in the transduction pathways of this allosteric motor, Structure, 15, (2007), 553-564, DOI 10.1016/j.str.2007.03.010.

9 Málnási-Csizmadia A, Kovács M, Emerging complex pathways of the actomyosin powerstroke, Trends in Biochemical Sciences, 35, (2010), 684-690, DOI 10.1016/j.tibs.2010.07.012.

10 Kuhner S, Fischer S, Structural mechanism of the ATP-induced dissociation of rigor myosin from actin, Proc. Natl. Acad. Sci. (USA), 108, (2011), 7793-7798, DOI 10.1073/pnas.1018420108.

11 Huxley AF, Muscle structure and theories of contraction, Progr. Biophys. Biophys. Chem., 7, (1957), 255-318.

12 Eisenberg E, Hill TL, A cross-bridge model of muscle contraction, Prog. Biophys. Molec. Biol., 33, (1979), 55-82, DOI 10.1016/00796107(79)90025-7.

13 Bibó A, Károlyi Gy, Spatial and temporal separation in overdamped systems, Periodica Polytechnica - Civil Engineering, 54, (2010), 89-94, DOI 10.3311/pp.ci.2010-2.03.

14 Landau LD, Lifshitz EM, Pitaevskii LP, Statistical physics, Pergamon Press, New York, 1980.

15 Protein Data Bank, www.pdb.org. PDB code of the conformation: 2Mys. Data obtained in 2012.

16 Yanagida T, Kitamura K, Tanaka H, Iwane AH, Esaki S, Single molecule analysis of the actomyosin motor, Curr. Opin. Cell Biol., 12, (2000), 20-25, DOI 10.1016/S0955-0674(99)00052-6. 